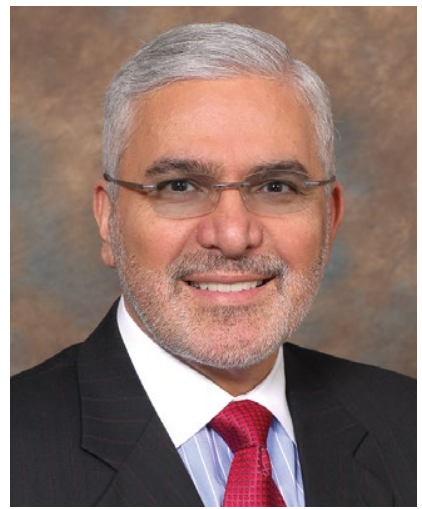

Henry A. Nasrallah, MD Editor-in-Chief

doi: 10.12788/cp.0161

\section{Measuring the}

molecular components of the CSF may help reveal granular clues to the neurobiology of psychiatric disorders

\title{
Needed: More studies of CSF molecular biomarkers in psychiatric disorders
}

Psychiatry and neurology are the brain's twin medical disciplines. Unlike neurologic brain disorders, where localizing the "lesion" is a primary objective, psychiatric brain disorders are much more subtle, with no "gross" lesions but numerous cellular and molecular pathologies within neural circuits.

Measuring the molecular components of the cerebrospinal fluid (CSF), the glorious "sewage system" of the brain, may help reveal granular clues to the neurobiology of psychiatric disorders.

Mental illnesses involve the disruption of brain structures and functions in a diffuse manner across the cortex. Abnormal neuroplasticity has been implicated in several major psychiatric disorders. Examples include hypoplasia of the hippocampus in major depressive disorder and cortical thinning/dysplasia in schizophrenia. Reductions of neurotropic factors such as nerve growth factor or brainderived neurotropic factor have been reported in mood and psychotic disorders, and appear to correlate with neuroplasticity changes.

Recent advances in psychiatric neuroscience have provided many clues to the pathophysiology of psychopathological conditions, including neuroinflammation, oxidative stress, apoptosis, impaired energy metabolism, abnormal metabolomics and lipidomics, and hypo- and hyperfunction of various neurotransmitters systems (especially glutamate $\mathrm{N}$-methyl-D-aspartate receptors, dopamine D2 receptors, serotonin 5-HT2A receptors, and gamma-aminobutyric acid receptors). Gray matter neurodegenerative changes as well as myelin disintegration in various psychiatric disorders point to significant neuropil and white matter pathologies across major psychiatric disorders. Dysfunctional glial cells (astroglia, microglia, and oligodendroglia) have also emerged as a central abnormality in psychiatric disorders. Permeability of the blood-brain barrier, and its adverse neural consequences, is also a fertile new area of research, reflecting how the brain of individuals with psychiatric illness may be vulnerable to peripheral inflammation and diseases.

Thus, psychiatric research should focus on exploring and detecting molecular signatures (ie, biomarkers) of psychiatric disorders, including biomarkers of axonal and synaptic damage, glial activation, and oxidative stress. This is especially critical given the extensive heterogeneity of schizophrenia and mood and anxiety disorders. The CSF is a vastly 
continued from page 6

\section{Table}

Editorial Staff

EDITOR Jeff Bauer

SENIOR EDITOR Sathya Achia Abraham

ASSISTANT EDITOR Matthew Wyles

WEB EDITOR Kathryn Wighton

Art \& Production Staff

CREATIVE DIRECTOR Louise Koenig

ART DIRECTOR Pat Fopma

DIRECTOR, JOURNAL MANUFACTURING Michael Wendt

PRODUCTION MANAGER Donna Pituras

\section{Publishing Staff}

PUBLISHER Sharon Finch

DIRECTOR EBUSINESS DEVELOPMENT

Alison Paton

SENIOR DIRECTOR OF SALES

Tim LaPella

Editor-in-Chief Emeritus

James Randolph Hillard, MD

Frontline Medical Communications

\section{VP, SALES Mike Guire}

VP, DIGITAL CONTENT \& STRATEGY Amy Pfeiffer

PRESIDENT, CUSTOM SOLUTIONS JoAnn Wahl

CIRCULATION DIRECTOR Jared Sonners

In affiliation with Global Academy for Medical Education, LLC

PRESIDENT David J. Small, MBA

\section{FRONTLINE MDedge}

7 Century Drive, Suite 302

Parsippany, NJ 07054

Tel: (973) 206-3434

Fax: (973) 206-9378

www.frontlinemedcom.com

Subscription Inquiries:

subscriptions@mdedge.com

Published through an educational partnership with

\section{lC CINCINNATI}

8 Current Psychiatry

8 August 2021

\section{Cerebrospinal fluid findings in psychiatric patients}

\begin{tabular}{|c|}
\hline Schizophrenia \\
\hline$\uparrow$ Total protein \\
\hline$\uparrow \mathrm{CSF} /$ serum albumin ratio \\
\hline$\downarrow$ Brain-derived neurotrophic factor (BDNF) \\
\hline$\downarrow$ Nerve growth factor \\
\hline$\uparrow$ Production of immunoglobulins in subgroups \\
\hline$\uparrow$ Blood-brain barrier permeability of inflammatory molecules \\
\hline$\uparrow$ CSF neurofilament light chain, a biomarker of neurodegeneration \\
\hline$\uparrow$ CSF cell counts ( 5 to $8 / \mathrm{mm}^{3}$ ) \\
\hline$\uparrow$ Proinflammatory biomarkers interleukin-6 and interleukin-8 \\
\hline $\begin{array}{l}\downarrow \text { Neuronal cell adhesion molecules (NCAM), correlating negatively with symptoms } \\
\text { (NCAM is vital for neuroplasticity) }\end{array}$ \\
\hline $\begin{array}{l}\downarrow \text { Glial cell line-derived neurotrophic factor (GDNF), and especially lower with longer } \\
\text { duration of illness }\end{array}$ \\
\hline$\uparrow$ BDNF and GDNF with antipsychotic treatment \\
\hline Alteration in glutamate homeostasis \\
\hline Alteration in cannabinoid metabolism \\
\hline Mood disorders \\
\hline$\uparrow$ Neopterin (released from astroglia) in a subgroup \\
\hline$\downarrow$ NCAM (correlated negatively with depressive symptom scores) \\
\hline$\uparrow$ GDNF in mania \\
\hline$\uparrow$ Proinflammatory biomarkers interleukin- 6 and interleukin-8 \\
\hline$\uparrow$ S100B (a calcium-binding protein in glia) \\
\hline 42 differentially regulated proteins in major depressive disorder \\
\hline Immune reaction reflected by oligoclonal immunoglobulin $\mathrm{G}$ in a subgroup \\
\hline Viral, bacterial, or autoimmune CNS involvement in a subgroup \\
\hline Mild encephalitis path mechanism in a subgroup \\
\hline
\end{tabular}

Source: References 1-9

unexploited substrate for discovering molecular biomarkers that will pave the way to precision psychiatry, and possibly open the door for completely new therapeutic strategies to tackle the most challenging neuropsychiatric disorders.

\section{A role for CSF analysis}

It's quite puzzling why acute psychiatric episodes of schizophrenia, bipolar disorder, major depressive disorder, or panic attacks are not routinely assessed with a spinal tap, in conjunction with other brain measures such as neuroimaging (morphology, spectroscopy, cerebral blood flow, and diffusion tensor imaging) as well as a comprehensive neurocognitive examination and neurophysiological tests such as pre-pulse inhibition, mismatch negativity, and P-50, N-10, and P-300 evoked potentials. Combining CSF analysis with all those measures may help us stratify the spectra of psychosis, depression, and anxiety, as well as posttraumatic stress 
disorder and obsessive-compulsive disorder, into unique biotypes with overlapping clinical phenotypes and specific treatment approaches.

There are relatively few published CSF studies in psychiatric patients (mostly schizophrenia and bipolar and depressive disorders). The Table $^{1-9}$ (page 8) shows some of those findings. More than 365 biomarkers have been reported in schizophrenia, most of them in serum and tissue. ${ }^{10}$ However, none of them can be used for diagnostic purposes because schizophrenia is a syndrome comprised of several hundred different diseases (biotypes) that have similar clinical symptoms. Many of the serum and tissue biomarkers have not been studied in CSF, and they must if advances in the neurobiology and treatment of the psychotic and mood spectra are to be achieved. And adapting the CSF biomarkers described in neurologic disorders such as multiple sclerosis ${ }^{11}$ to schizophrenia and bipolar disorder (which also have well-established myelin pathologies) may yield a trove of neurobiologic findings.

If CSF studies eventually prove to be very useful for identifying subtypes for diagnosis and treatment, psychiatrists do not have to do the lumbar puncture themselves, but may refer patients to a "spinal tap" laboratory, just as they refer patients to a phlebotomy laboratory for routine blood tests. The adoption of CSF assessment in psychiatry will solidify its status as a clinical neuroscience, like its sister, neurology.

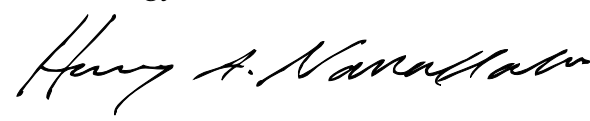

Henry A. Nasrallah, MD

Editor-in-Chief

\section{References}

1. Vasic N, Connemann BJ, Wolf RC, et al. Cerebrospinal fluid biomarker candidates of schizophrenia: where do we stand? Eur Arch Psychiatry Clin Neurosci. 2012;262(5):375-391.

2. Pollak TA, Drndarski S, Stone JM, et al. The bloodbrain barrier in psychosis. Lancet Psychiatry. 2018;5(1):79-92.

3. Katisko K, Cajanus A, Jääskeläinen $\mathrm{O}$, et al. Serum neurofilament light chain is a discriminative biomarker between frontotemporal lobar degeneration and primary psychiatric disorders. J Neurol. 2020;267(1):162-167.

4. Bechter K, Reiber H, Herzog S, et al. Cerebrospinal fluid analysis in affective and schizophrenic spectrum disorders: identification of subgroups with immune responses and blood-CSF barrier dysfunction. J Psychiatr Res. 2010;44(5):321-330.

5. Hidese S, Hattori K, Sasayama D, et al. Cerebrospinal fluid neural cell adhesion molecule levels and their correlation with clinical variables in patients with schizophrenia, bipolar disorder, and major depressive disorder. Prog Neuropsychopharmacol Biol Psychiatry. 2017;76:12-18.

6. Tunca Z, Kivırcık Akdede B, Özerdem A, et al. Diverse glial cell line-derived neurotrophic factor (GDNF) support between mania and schizophrenia: a comparative study in four major psychiatric disorders. Eur Psychiatry. 2015;30(2):198-204.

7. Al Shweiki MR, Oeckl P, Steinacker $\mathrm{P}$, et al. Major depressive disorder: insight into candidate cerebrospinal fluid protein biomarkers from proteomics studies. Expert Rev Proteomics. 2017; 14(6):499-514.

8. Kroksmark H, Vinberg M. Does S100B have a potential role in affective disorders? A literature review. Nord J Psychiatry. 2018;72(7):462-470.

9. Orlovska-Waast S, Köhler-Forsberg O, Brix SW, et al. Cerebrospinal fluid markers of inflammation and infections in schizophrenia and affective disorders: a systematic review and meta-analysis. Mol Psychiatry. 2019;24(6):869-887.

10. Nasrallah HA. Lab tests for psychiatric disorders: few clinicians are aware of them. Current Psychiatry. 2013;12(2):5-7.

11. Porter L, Shoushtarizadeh A, Jelinek GA, et al. Metabolomic biomarkers of multiple sclerosis: a systematic review. Front Mol Biosci. 2020;7:574133. doi: $10.3389 /$ fmolb.2020.574133
The adoption of CSF assessment in psychiatry will solidify its status as a clinical neuroscience, like its sister, neurology 